\title{
Numerical Vibro-Acoustic Analysis of a Ship Compartment Structure
}

\author{
Mihaela Barcan $^{1}$ (Savin), Ionel Chirica ${ }^{1, *}$ and Elena-Felicia Beznea ${ }^{1}$ \\ 1,'Dunărea de Jos” University of Galati, Faculty of Engineering, Domnească Street 47, Romania
}

\begin{abstract}
This paper investigates the vibro-acoustic problems of an elastic compartment structure (wheel house) belonging to a ship, coupled to the enclosed air. The excitations from the structural walls of the room by the sound source cause vibrations throughout the system and noise within the acoustic domain. Due to the coupling between the structural vibrations and acoustic pressure field, these systems are typically referred to as vibroacoustic systems. The acoustic excitations and vibrations on the structural walls of a ship structure, however, are significantly larger and have a much more significant effect to the structural integrity of the ship. In order to determine whether coupled responses are needed, three frequency analyses have been performed: calculus of the first 5 natural frequencies of the fluid separately, the structure separately, and the fluid-structure coupled system. The wall thickness of the room walls is of $5 \mathrm{~mm}$ and made of layered composite. The stress analysis of the model at a highly participating structural mode is performed.
\end{abstract}

\section{Introduction}

The noise is one of the most important pollution parameter for many industries. In ship transport, especially in the case of passengers ships the interior noise is a criteria imposed by classification societies rules. To reduce time and effort for the ship interior noise analysis, it is usual to perform the related work during the design stage.

The transmission phenomena and sources of ship noise depend on the frequency range. The most important noise is the structure-borne type in the case of the low and medium frequency range. The structure vibrations radiate sound so into the interior as well as into the exterior of the ship. The ship structure vibrations can be caused by various sources, such as engines, generators, rotating systems, interaction of water from propeller that hits the aft part of the ship. The phenomenon of interior noise represents the fluid-structure interaction (coupling between ship structural vibrations and air pressure fluctuations in the interior of a compartment), when the energy exchange between the acoustic sub-system and structural sub-system is performed. Because the interior noise dynamic behaviour of each of the both sub-systems is affected by the behaviour of the other one, the reciprocal actions between air and ship hull structure is transformed into a complex phenomenon.

\footnotetext{
${ }^{1}$ Corresponding author: ionel.chirica@ugal.ro
} 
The vibro-acoustic analysis is very important especially in the case of elastic structures. The designers work to avoid resonances (or near resonances) that produce large dynamic displacements of the elastic structures and of course variations of pressures of surrounding air (noise).

The reduction of acoustic noise levels and environmental sound has been established in the international norms in shipbuilding which are used to quantify the noise generated from acoustic sources.

In [1] analytical, finite element analysis, associated with experimental studies are presented and compared. The analytical method is based on the interior acoustic pressure that is developed function of the terms of structural and acoustic normal modes.

In [2] problem of damping at the interface fluid-structure is treating. The additional coupled field, named normal fluid displacement field, is concerning an elastic structure containing an inviscid, compressible and barotropic fluid. So, various damping models on the interface can be used in the variational formulation.

In [3] the design sensitivity analysis of a structural-acoustic problem is presented. The frequency-response analysis is performed to solve the dynamic behavior of a car structure. The problem is decoupled since the boundary element method is used to solve the pressure response of an interior domain.

\section{Sound theory}

The sound wave is propagating as longitudinal waves that concern successive compression and rarefaction of the air, considered as an elastic medium (see Fig. 1), [4]. The sound pressure is considered as being negligible compared with pressure fluctuations around atmospheric pressure.

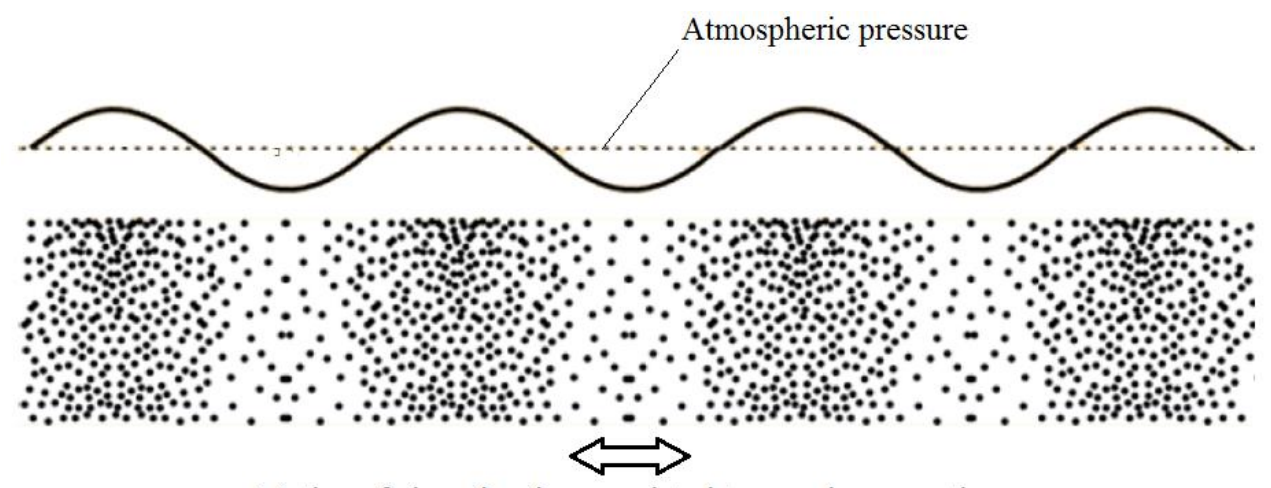

Motion of air molecules associated to sound propagation

Fig. 1. Sound wave propagation

In the structural acoustics analysis, the interaction of vibrating structures with adjacent fluid is studied. During this phenomenon, the radiated or scattered sound is performed. The structure having relative motion starts to vibrate and therefore pressure fluctuations in surrounding medium are producing. Sometimes, pressure fluctuations are causing vibration of adjacent structures. The pressure fluctuations are negligible versus acoustic waves. The common sound sources are structures loaded by transient or oscillatory loads. Due to the presence of the fluid medium, vibration characteristics of the structure can be significantly changed. The acoustic wave moving is performed by structural velocity (only normal component of structural surface velocity). During structural acoustic the fluid is considered to be ideal, non-viscous and without any shear layer's effect. 
Sound wave propagating through a solid medium (like structure) is called structural-borne sound and if it is propagating through the air medium it is called air-borne sound [4]. The airborne sound is caused by an external source and propagates further into the structures like ship compartment panels (the floor, wall panels, decks etc.). The structure-borne sound is produced by the mechanical vibrations that are propagating through the ship structure.

In the paper, structural acoustic analysis has been done in ANSYS using ANSYS/ Multiphysics and ANSYS/Mechanical programmes. In the acoustic analysis the modeling of structure field and fluid field, depending on external or internal problem, is performed with appropriate elements types. According to the connections the boundary conditions and loads are applied. The solving of the problem is made by using a valid method.

Acoustic model consists of four systems: structural field (using SHELL181 element type), fluid field (using FLUID30, that are 3D finite acoustic elements), fluid-structure interfaces and infinite acoustic field (using FLUID130, which are the infinite acoustic elements).

This study is focused to develop a methodology based on FE formulation for structural acoustic analysis of the wheel house structure made of steel plates surrounded by air medium in the ship compartment.

In acoustical fluid-structure interaction problems, the acoustic wave equation and the structural dynamics equation are used. These two equations have to be coupled to each other. The acoustic wave equation is given by equation

$$
\frac{1}{c^{2}} \frac{\partial^{2} p}{\partial t^{2}}-\nabla^{2} p=0
$$

where:

$c$ is the speed of sound in fluid field, being determined by equation o

$$
c=\sqrt{\frac{k}{\rho_{o}}}
$$

$\rho_{o}$ is the mean density of the fluid;

$k$ is bulk modulus of the fluid;

$p$ is the acoustic pressure;

$t$ is time.

By using the FEM theory, the matrix equation is written as the follows

$$
\boldsymbol{m}_{e}^{p} \ddot{\boldsymbol{p}}_{e}+\boldsymbol{k}_{e}^{p} \boldsymbol{p}_{e}=\mathbf{0}
$$

In equation (3), $\boldsymbol{p}_{e}$ is nodal pressure vector. The matrices from the equation are:

$\boldsymbol{m}_{e}^{p}=\frac{1}{c^{2}} \int_{V} \boldsymbol{N}^{T} \boldsymbol{N} d V$ is the inertia matrix,

$\boldsymbol{k}_{e}^{p}=\int_{V} \boldsymbol{B}^{T} \boldsymbol{B} d V$ is the stiffness matrix,

$\boldsymbol{B}=\boldsymbol{L} \boldsymbol{N}^{T}$ is the strain variation approach functions matrix,

$\boldsymbol{L}=\nabla()$ 
In the fluid-structure interaction problem, the natural boundary condition along the interface has to be included. The fluid momentum equations give the following relationship between the pressure gradient of the fluid and the normal acceleration of the structure at the fluid-structure interface

$$
\boldsymbol{n} \nabla \boldsymbol{p}=-\rho_{0} \boldsymbol{n} \frac{\partial^{2} \boldsymbol{u}}{\partial t^{2}}
$$

The most used essential boundary conditions are:

- On the free surface of fluid cavity, $p=0$,

- On solid boundary of the cavity,

- For rigid boundary, $\ddot{u}_{n}=0$,

$$
\frac{\partial p}{\partial n}=-\rho \ddot{u}_{n}
$$

where $n$ is the normal vector to the boundary.

By including the fluid-structure interface condition into the wave equation and writing it in finite-element matrix formulation, equation (3) becomes

$$
\boldsymbol{m}_{e}^{p} \ddot{\boldsymbol{p}}_{e}+\boldsymbol{k}_{e}^{p} \boldsymbol{p}_{e}+\rho_{0} \boldsymbol{R}^{T} \ddot{\boldsymbol{u}}_{e}=\boldsymbol{0}
$$

where

$\boldsymbol{R}^{T}=\int_{S} \boldsymbol{N}^{T} \boldsymbol{N} \boldsymbol{N}^{\prime T} d S$ is the fluid structure coupling mass matrix,

$N^{\prime}$ is the matrix of element approach functions of the displacements components $u_{x}, u_{y}, u_{z}$ obtained from the structural element,

$\boldsymbol{u}_{\boldsymbol{e}}$ is the vector of nodal displacements (index $\boldsymbol{e}$ means element),

$S$ is the surface where the derivative of pressure normal to the surface is applied.

When the dissipation of energy due to damping does exists, the term of dissipation has to be added. In this case, the damping is considered a proportionally to the displacement speed. So, the equation (4) becomes

$$
\boldsymbol{m}_{e}^{p} \ddot{\boldsymbol{p}}_{e}+\boldsymbol{c}_{e}^{p} \dot{\boldsymbol{p}}_{e}+\boldsymbol{k}_{e}^{p} \boldsymbol{p}_{e}+\rho_{0} \boldsymbol{R}^{T} \ddot{\boldsymbol{u}}_{e}=\mathbf{0}
$$

where the damping matrix of fluid is determined with the equation

$$
c_{e}^{p}=\frac{\beta}{c} \int_{S} N N^{T} d S
$$

where $\beta$ is the boundary absorption coefficient.

The fluid pressure load acting at the fluid-structure interface has to be added to the structural dynamic equation and gives the matrix equation

$$
\boldsymbol{m}_{e} \ddot{\boldsymbol{u}}_{e}+\boldsymbol{c}_{e} \ddot{\boldsymbol{u}}_{e}+\boldsymbol{k}_{e} \boldsymbol{u}_{e}=\boldsymbol{F}_{e}+\boldsymbol{F}_{e}^{p r}
$$

In equation (6), $\boldsymbol{F}_{e}{ }^{p r}$ is the fluid pressure load vector at the interface, determined as in the following, performing the coupling between fluid parameter $\left(\boldsymbol{p}_{\boldsymbol{e}}\right)$ and structure parameter $(\boldsymbol{u} \boldsymbol{e})$. 


$$
\boldsymbol{F}_{e}^{p r}=\boldsymbol{R} \boldsymbol{p}_{e}
$$

Equations (5) and (6) describe the finite-element equations for the fluid-structure interaction problem and are written in assembled form as

$$
\left[\begin{array}{cc}
\boldsymbol{m}_{s} & \boldsymbol{0} \\
\boldsymbol{m}_{f s} & \boldsymbol{m}_{f}
\end{array}\right]\left[\begin{array}{l}
\ddot{\boldsymbol{u}} \\
\ddot{\boldsymbol{p}}
\end{array}\right]+\left[\begin{array}{cc}
\boldsymbol{c}_{s} & \boldsymbol{c}_{f s} \\
\boldsymbol{0} & \boldsymbol{c}_{f}
\end{array}\right]\left[\begin{array}{l}
\dot{\boldsymbol{u}} \\
\dot{\boldsymbol{p}}
\end{array}\right]+\left[\begin{array}{cc}
\boldsymbol{k}_{s} & \boldsymbol{k}_{f s} \\
\boldsymbol{0} & \boldsymbol{k}_{f}
\end{array}\right]\left[\begin{array}{c}
\boldsymbol{u} \\
\boldsymbol{p}
\end{array}\right]=\left[\begin{array}{c}
\boldsymbol{F}_{s} \\
\boldsymbol{0}
\end{array}\right]
$$

In equation (7) $\boldsymbol{m}_{f s}$ and $\boldsymbol{k}_{f s}$ are so-name inertia and respectivelly stiffness matrices of fluid structure interaction $(f s)$, determined with the equations

$$
\begin{gathered}
\boldsymbol{m}_{f s}=\rho_{0} \boldsymbol{R}^{T} \\
\boldsymbol{k}_{f s}=-\boldsymbol{R}
\end{gathered}
$$

Presence of matrix $\boldsymbol{R}$, causes the non-symmetric matrix equation equation (7). For solving, the equation can be converted it into a symmetric form using displacement potential function [5]. For this action, it is necessary to integrate pressure equation with time and to do the substitution. By rearranging the terms, the system (7) becomes now a symmetric one

$$
\left[\begin{array}{cc}
\boldsymbol{m}_{s} & \boldsymbol{0} \\
\boldsymbol{0} & \frac{1}{\rho} \boldsymbol{m}_{f}
\end{array}\right]\left[\begin{array}{c}
\ddot{\boldsymbol{u}} \\
\ddot{\boldsymbol{q}}
\end{array}\right]+\left[\begin{array}{cc}
\boldsymbol{c}_{S} & -\boldsymbol{R}^{T} \\
\boldsymbol{R} & \frac{1}{\rho} \boldsymbol{c}_{f}
\end{array}\right]\left[\begin{array}{l}
\dot{\boldsymbol{u}} \\
\dot{\boldsymbol{q}}
\end{array}\right]+\left[\begin{array}{cc}
\boldsymbol{k}_{S} & \boldsymbol{k}_{f s} \\
\boldsymbol{0} & \boldsymbol{k}_{f}
\end{array}\right]\left[\begin{array}{c}
\boldsymbol{u} \\
\boldsymbol{q}
\end{array}\right]=\left[\begin{array}{c}
\boldsymbol{F}_{S} \\
\boldsymbol{0}
\end{array}\right]
$$

The coupling matrix $\boldsymbol{R}$ transferred from mass and stiffness parts to the damping part when the equation is switched to velocity potential as unknown.

In particular cases (low-frequency eigenvalue calculations), the fluid being incompressible $\left(\boldsymbol{m}_{f}=\boldsymbol{0}\right)$ and by neglecting damping effect $\left(\boldsymbol{c}_{f}=\boldsymbol{0}\right)$, the well-known eigenvalue problem is obtained

$$
\left(\boldsymbol{m}_{s}+\boldsymbol{m}_{a}\right) \ddot{\boldsymbol{u}}+\boldsymbol{k}_{s} \boldsymbol{u}=\boldsymbol{F}_{s}
$$

where $\boldsymbol{m}_{\boldsymbol{a}}$ is added mass, having the form

$$
\boldsymbol{m}_{a}=\rho \boldsymbol{R} \boldsymbol{k}_{f}^{-1} \boldsymbol{R}^{T}
$$

The case study performed in this paper is the analysis of coupling effects of the FE model. In some acoustic problems involving gases (air), only the uncoupled structural model response may be accurate enough. The coupled structural acoustic matrix depends primarily on the mass and stiffness of the components. In many cases when gases (air) are incorporated into the system, the most part of the coupled matrix has the structure contribution. Equation of motion of a freely vibrating vibro-acoustic system without damping, may be written as

$$
-\omega_{n}^{2}\left[\begin{array}{cc}
M_{s} & 0 \\
M_{f_{s}} & M_{a}
\end{array}\right]+\left[\begin{array}{cc}
K_{s} & K f_{s} \\
0 & K_{a}
\end{array}\right]\left[\begin{array}{l}
u \\
p
\end{array}\right]=\left[\begin{array}{l}
0 \\
0
\end{array}\right]
$$

where

$\omega_{\mathrm{n}}$ is natural frequency,

$u$ is vector of nodal displacements, 
$p$ is vector of nodal pressure,

$M_{s}$, is inertia matrix of the elastic structure,

$K_{s}$ is the stiffness matrix of the elastic structure,

$M_{a}$ is the inertia matrix of the acoustic cavity,

$K_{a}$ is the stiffness matrix of the acoustic cavity,

$M_{f s}$ is the coupled system mass matrix for the elastic structure,

$K_{f s}$ is the coupled system stiffness matrix for the elastic structure.

Solution of the equation (10) gives eigenvalues (natural frequencies) and eigenvectors (modal shapes) of the system.

\section{Finite element simulation model}

In order to determine whether coupled responses are needed, three model types of frequency analysis have been performed: calculus of the first 10 natural frequencies of the fluid separately, the structure separately and the fluid-structure coupled system.

Among the three models, the one for plate system structure is the least sensitive system to acoustic pressure. That is, the least effective coupling behaviour is present in plate system.

The stress analysis of the model at a highly participating structural mode has been performed. The stress concentration that appears in the walls are illustrated and discussed.

Vibration and acoustic analysis of wheel house compartment (made only of steel plates) was carried out in finite element simulation. Simulation model consist of structure field, fluid field and fluid-structure interface. Structure field has been modeled using SHELL181 element while fluid volume is modeled using FLUID30. Fluid-structure interface has been defined on common nodes for structure field and fluid field. The simulation model in which all compartment plates, of $5 \mathrm{~mm}$ thickness, are surrounded by fluid domain have been done in ANSYS.

In table 1, the values of the first 5 natural frequencies of the fluid-structure coupled system are shown. Due to the coupling, the values are greater than the natural frequencies of the structure separately.

Table 1. Natural frequencies

\begin{tabular}{|c|c|}
\hline Mode No. & Natural frequency $[\mathrm{Hz}]$ \\
\hline 1 & 78.542 \\
\hline 2 & 125.22 \\
\hline 3 & 136.37 \\
\hline 4 & 149.98 \\
\hline 5 & 165.46 \\
\hline
\end{tabular}

Fig. 2 shows pressure variation obtained by simulation in ANSYS. As it is seen, after two-three periods, the pressure oscillation is stabilized. 


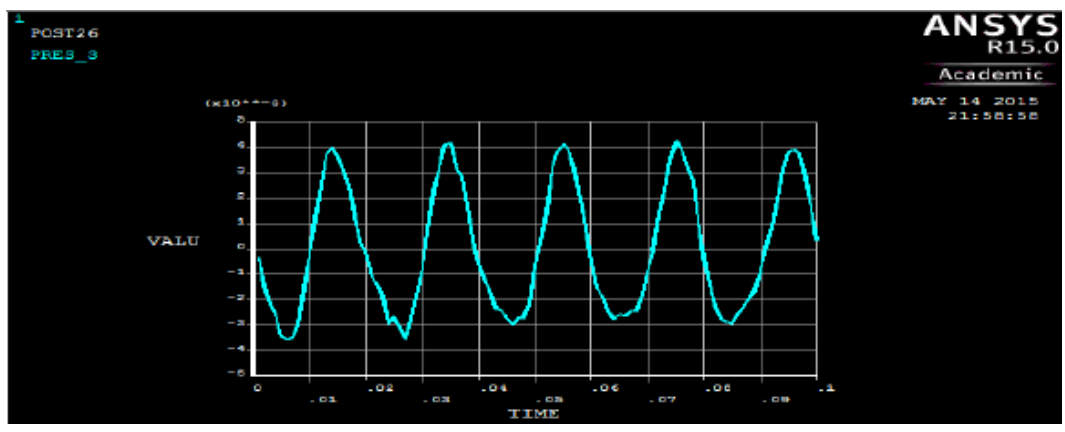

Fig. 2. Pressure variations of the compartment in ANSYS.

Fig. 3 shows modes shapes for the acoustic cavity of the ship compartment (pressure distribution).

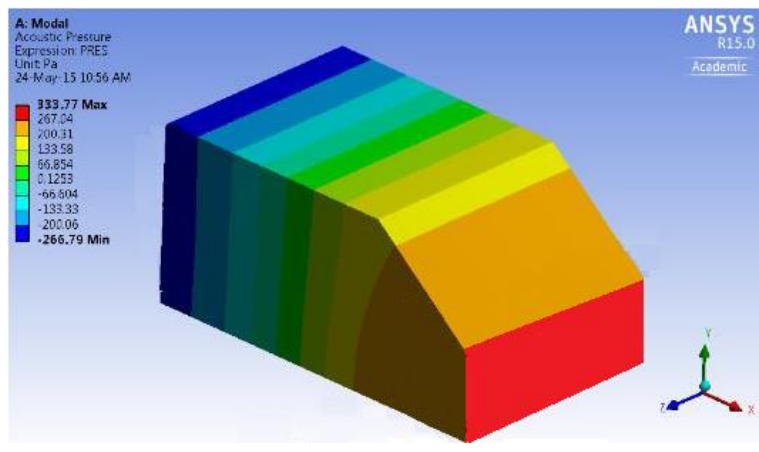

Fig. 3. Pressure distribution due to wheel house compartment vibration

\section{Conclusions}

The developed experimental model for vibro-acoustic analysis would be useful for analysis of compartment structures under different boundary and loading conditions. It is observed that the present developed FE model in ANSYS environment is also capable to solve any fluid-structure interaction problem. And hence, the present analysis would be useful for the practical design of the structure. In order to determine whether coupled responses are needed, three frequency analyses have been performed: calculus of the first 5 natural frequencies of the fluid separately, the structure separately, and the fluid-structure coupled system. The stress concentration that appears in the room walls are illustrated and discussed.

The paper has been supported by Doctoral School of Mechanical and Industrial Engineering from University Dunarea de Jos of Galati, Romania.

\section{References}

1. V.B. Georgiev, V.V. Krylov and R.E.T.B. Winward, J. Low Freq. Noise, Vibr. and Act. Control, 25, 2, (2006) 
2. J.F. Deü, W. Larbi, R. Ohayon, Comput Methods Appl Mech Eng, Elsevier, 197, 51-52, (2008)

3. N.H. Kim, Jun Dong, Kyung K. Choi, N. Vlahopoulos, Z.-D. Ma, M.P. Castanierd, C. Pierre, J Sound Vib 263, (2003)

4. S. S. Bankar, Vibration and Acoustic Analysis of Laminated Composite Plate, Master thesis, Rourkela Odisha (India), (2015)

5. G.C. Everstine, J Sound Vib, 79, (1981) 